\title{
Mejora Continua del Servicio al Cliente Mediante ServQual y Red de Petri en un Restaurante de Santa Marta, Colombia
}

\author{
Edwin Causado-Rodriguez ${ }^{1 *}$, Arturo N. Charris ${ }^{2}$ y Edwin A. Guerrero ${ }^{2}$ \\ (1) Universidad del Magdalena, Facultad de Ingeniería, Programa de Ingeniería Industrial, Cra 32 No. 22 - \\ 08 San Pedro Alejandrino, Santa Marta, Colombia. (e-mail: ecausado@unimagdalena.edu.co) \\ (2) Universidad del Magdalena, Facultad de Ciencias Empresariales y Económicas, Cra 32 No. 22 - 08 San \\ Pedro Alejandrino, Santa Marta, Colombia. (e-mail: acharris@unimagdalena.edu.co; \\ guerrero@unimagdalena.edu.co)
}

* Autor a quien debe ser dirigida la correspondencia

Recibido Jul. 26, 2018; Aceptado Oct. 11, 2018; Versión final Nov. 19, 2018, Publicado Abr. 2019

\begin{abstract}
Resumen
Se aplica el Método ServQual en el servicio de restaurantes con el fin de obtener una medida de la calidad del servicio. El procedimiento se complementa con el método Red de Petri, con el fin de evaluar el componente de capacidad de respuesta, en la prestación de un servicio en el sector de restaurantes. Metodológicamente, la mejora continua se evaluó mediante encuestas de satisfacción del cliente. Además, se registraron tiempos de pedidos, preparación, domicilios y llegadas de clientes, verificados por medio de herramientas informáticas como Input Analyser y Microsoft Excel. Posteriormente, se validó experimentalmente el diseño en simulación en redes de Petri, mediante Visual Basic. Con los resultados obtenidos se lograron mejoras del proceso, que se traducen en recomendaciones, en temas como: incremento en el personal del restaurante, mayor número de clientes atendidos, mejoras en la eficiencia y eficacia del servicio prestado.
\end{abstract}

\section{Continuous Improvement of Customer Service Through ServQual and Petri Network in Santa Marta Restaurant - Colombia}

\begin{abstract}
The ServQual Method was applied in the restaurant service with the aim of obtaining a measure of the quality of the service. The method was complemented using Petri Network method, in order to evaluate the response capacity component, in the provision of a service in the restaurant sector. Methodologically, continuous improvement was evaluated through customer satisfaction surveys. Also, order preparations, domiciles, and customer arrivals times, were registered and computer tools such as Input Analyzer and Microsoft Excel were employed. Subsequently, the design was validated experimentally by simulation using Petri nets with Visual Basic. With the results obtained, process improvements were achieved, which resulted in recommendations on topics such as: increase in restaurant staff, higher number of clients served, improvements in the efficiency and effectiveness of the service provided.
\end{abstract}

Keywords: customer satisfaction; ServQual method; Petri nets; continuous improvement 


\section{INTRODUCCIÓN}

Los negocios de preparación de alimentos y bebidas, representan una oportunidad de éxito empresarial, si se aplican estrategias eficaces para el ofrecimiento de productos diferenciados y de calidad (Fuentes-Blanco y Moliner-Velázquez, 2014; Schmal y Olave, 2014). Desafortunadamente muchos propietarios y administradores de restaurantes, dejan de lado la satisfacción de las expectativas de los clientes respecto al servicio prestado, por concentrarse, entre otros aspectos, en la calidad del producto, la financiación del negocio o en actividades básicas de la operación de la empresa, inadvirtiendo que el cliente busca algo más que un buen producto. En tal sentido cabe destacar, que el servicio al cliente es determinante para el consumidor (Kim y Lough, 2007), e implica aspectos tales como: el tiempo que debe esperar para ser atendido, la amabilidad del empleado al atenderle, la pulcritud o higiene de la infraestructura, el mobiliario y los trabajadores; así como la disponibilidad de horarios convenientes (Guzman-Lopez y Carcamo-Solis, 2014; Yu-qiang y Jun-jia, 2011). Diversos tratadistas ratificaron la importancia de la calidad del servicio, al argumentar que ha venido reconociéndose cada vez más como un determinante de la eficiencia y la eficacia en las operaciones comerciales (Cabana et al, 2015; Parasuraman et al, 1994; Parasuraman et al, 1991b). A su vez, desde la perspectiva del comportamiento del consumidor, también se sugiere que la calidad tiene incidencia importante en las intenciones de compra de los consumidores a través del papel mediador de las percepciones de valor asociadas a los productos y servicios (Du y Tang, 2014; Silva et al, 2014).

No obstante lo anterior, la conceptualización y medición de la calidad del servicio han permanecido típicamente sub-estudiadas (López y Serrano, 2001; Parasuraman et al, 1991a). Precisamente, han sido los esfuerzos en el área de marketing de servicios lo que ha permitido superar esta deficiencia, al desarrollar un instrumento de medición integral conocido como ServQual, que mide la percepción de la calidad del servicio consumido por un cliente, y que además puede complementarse con otras herramientas como el ciclo del servicio, el triángulo del servicio, la teoría de colas o la Red de Petri, como es el caso de esta investigación y que además representa su componente diferenciador. En concreto, en el caso del restaurante en la ciudad de Santa Marta - Colombia, los clientes han venido manifestando quejas respecto a la calidad del servicio recibido, situación que preocupa a la gerencia debido a que está repercutiendo en los ingresos y la participación de mercado de la empresa, haciéndose perentorio implementar acciones correctivas. Entre los factores más influyentes en la insatisfacción de sus clientes, resaltan problemáticas tradicionales como la falta de amabilidad del mesero, la demora en la toma de los pedidos, no tener las mesas limpias a tiempo, así como los retrasos en la preparación de los alimentos (Law, A.K, Hui, Y.V, Zhao, X., 2004). Consecuencia de lo anterior, en este artículo se propone el diseño de estrategias para la mejora continua de la calidad y del servicio de atención al cliente del restaurante en la ciudad de Santa Marta - Colombia, utilizando los métodos ServQual y Redes de Petri, luego de diagnosticar el nivel de satisfacción del cliente y priorizar acciones que favorezcan la optimación de la atención.

\section{MARCO TEORICO}

La industria de servicios empezó a ser reconocida desde la década de 1930, cuando Fisher (1935) institucionalizó el término "sector terciario". Aunque poco se había escrito sobre la calidad del servicio hasta la década de 1970, tan pronto empezó a hacerse visible su vínculo con las experiencias vividas del cliente durante la prestación del servicio, se allanó el camino para iniciar investigaciones respecto a su conceptualización y medición. En el marco de esa dinámica, desde entonces se vienen llevando a cabo importantes investigaciones sobre la calidad de los servicios (Rojas-Ríos et al, 2016). No obstante Parasuraman et al. (1988:1985), aclararon que inicialmente hubo limitantes al tratar de definir y modelar la calidad en las industrias de servicios, debido a dificultades en el constructo del concepto, al momento de delimitar y medir dada su intangibilidad. Pese a ello, paulatinamente se ha venido configurando una larga lista de investigadores en la temática de calidad del servicio, tales como Causado-Rodriguez, et al (2015); CallejasCuervo, et al (2014), Causado-Rodriguez, et al (2008), Cook (1999), quienes reconocen la característica intangible de los servicios, asumiéndolos en su mayoría como actuaciones o experiencias vividas, en contraste con los bienes tangibles que pueden ser poseídos.

Así las cosas, es de anotar que la intangibilidad de los servicios implica un alto grado de dificultad para establecer especificaciones precisas respecto a criterios de uniformidad en la calidad, lo cual dificulta tanto a gerentes como al personal y clientes la posibilidad de poder medir, probar o verificar la calidad de los servicios (Castillo y Torres, 2013; Laguna y Palacios, 2008; Babakus y Boller, 1992). Por lo tanto, para este estudio y para la demostración de la utilidad del respectivo modelo que lo constituye, resulta imperativo aplicar un instrumento válido y confiable que permita medir la calidad del servicio en un restaurante, como es el caso del restaurante en la ciudad de Santa Marta - Colombia, con el fin de captar la percepción de los patrones tanto tangibles como intangibles del servicio prestado, tales como: las instalaciones físicas (elementos tangibles), fiabilidad, capacidad de respuesta, seguridad y empatía; todos elementos existentes en el constructo del modelo ServQual. 


\section{Modelo ServQual}

El modelo ServQual, representa uno de los principales resultados de la investigación programática iniciada por Parasuraman et al. (1985:1988). Los modelos conceptuales desarrollados por estos investigadores, definen la calidad del servicio como la principal variable de resultado (Parasuraman et al; 1994; Parasuraman et al; 1991; Parasuraman et al; 1988; y Parasuraman et al., 1985). Dicho modelo, contiene una serie de ítems destinados a medir los niveles de servicio esperado por el cliente, a lo cual se le denomina expectativas. Mediante esos mismos ítems coincidentes, se mide el nivel percibido de servicio proporcionado (servicio real) por una organización, designados estos como percepciones. Finalmente, la calidad del servicio se obtiene al medir la diferencia de puntuaciones entre ítems correspondientes, o lo que es lo mismo, al establecer la diferencia entre expectativas y percepciones.

Según la conceptualización de Parasuraman et al. (1988), define la calidad del servicio, como un constructo de cinco dimensiones consistentes, a saber: elementos tangibles, confiabilidad, capacidad de respuesta, seguridad y empatía; dimensiones que a su vez, están constituidas por 22 ítems, a través de los cuales se alimenta el modelo y se determina el gap o brecha entre las dos valoraciones (es decir, entre lo que el cliente espera del servicio y lo que realmente percibe), con el objeto de facilitar la puesta en marcha; sistemáticamente de acciones correctivas que mejoren la calidad del servicio (McCollin et al, 2011; Yu-qiang, y Jun-Jia, 2011; López y Serrano, 2001; Lee y Hing, 1995). En suma, el modelo ServQual ofrece un mapa de la situación respecto a la calidad en la prestación del servicio y los aspectos que verdaderamente valora el cliente al momento de acceder a dicho servicio, como es el caso del restaurante objeto de estudio, lo cual en últimas, debe incidir en la captación de un mayor número de clientes, mantener los existentes y mejorar los niveles de rentabilidad para la organización (Cabana et al, 2015; Du y Tang, 2014; Tan et al, 2009).

\section{Red de Petri}

Por su parte, la Red de Petri, permite modelar procesos de flujos de trabajo y detectar errores de diseño estructural o problemas de comportamiento, en el manejo de las capacidades de la empresa (Tan et al, 2009; Adam et al, 1998; Van Der Aalst, 1994:1996). Dicha red, a su vez, constituye una poderosa herramienta que permite describir y analizar el comportamiento de los sistemas de eventos discretos y, especialmente evaluar procesos complejos tales como representación y análisis de procesos concurrentes (Liu, 2009; Jensen, 1996; Teas, 1993).

La Red de Petri clásica, fue originada por Carl Adam Petri en los años sesenta (Petri, 1962). Desde entonces se han utilizado las Redes de Petri, para modelar y analizar todo tipo de procesos con aplicaciones que van desde protocolos, hardware y sistemas integrados hasta sistemas flexibles de fabricación, interacción del usuario y procesos, análisis en eficiencia de servicios. En las dos últimas décadas se ha extendido la Red de Petri clásica con color, tiempo y jerarquía (Tan et al, 2009; Jensen, 1996; Van Der Aalst, 1994). Estas extensiones facilitan la modelación de procesos complejos donde los datos y el tiempo son factores importantes. De esta manera, las Redes de Petri son un grafo orientado, el cual se encuentra conformado por los elementos mostrados en la Tabla 1. En la tabla 1 se evidencia que los puntos denominados tokens, se mueven de un sitio a otro de acuerdo a una regla según la cual, pueden pasar a través de una transición solamente cuando un pulso de reloj y todas las flechas que apuntan a la transición emanan de sitios que contienen tokens.

Tabla 1: Propiedades de los elementos de la Red de Petri

\begin{tabular}{|c|l|}
\hline Elementos & \multicolumn{1}{|c|}{ Propiedades } \\
\hline Plazas & $\begin{array}{l}\text { Nombre del elemento (Almacén, Bodega, Maquina, Materia Prima, Operación, Trabajador, Mesa de } \\
\text { Trabajo, etc.). Contiene la capacidad de la Plaza, es decir la cantidad de Tokens que podrá admitir la } \\
\text { plaza. }\end{array}$ \\
\hline \begin{tabular}{c|} 
Transiciones \\
\hline Arcos
\end{tabular} & $\begin{array}{l}\text { Nombre de la transición (inicio, fin). Permite relacionar dos plazas. Como también se definen, como } \\
\text { acciones o eventos que causan cambios en los estados. Contiene el cambio de estado de una plaza y } \\
\text { otra (tiempos). }\end{array}$ \\
\hline Token & $\begin{array}{l}\text { Permiten unir las plazas y transiciones, y de esta manera armar el modelo de red, permitiendo indicar el } \\
\text { que podrán pasar al mismo tiempo por un arco). }\end{array}$ \\
\hline- & $\begin{array}{l}\text { Equivalen a una marca dentro del sistema; tales como, unidades de materia prima, productos en proceso } \\
\text { y producto terminado. }\end{array}$ \\
\hline
\end{tabular}




\section{MATERIALES Y METODOS}

La metodología aplicada en este estudio es de carácter descriptivo, explicativo y correlacional, dado que especifica cada una de las características de las variables de interés; esto es, calidad de servicio y satisfacción al cliente, determinando su nivel de influencia en la percepción del servicio esperado, versus el servicio recibido. Para la aplicación de las encuestas del modelo ServQual, se determinó el tamaño final de la muestra, tomando en cuenta la cantidad de clientes en promedio que asisten al restaurante durante un fin de semana por un mes. Dicha cantidad fue suministrada por el administrador del restaurante, dando como resultado un total de 440 clientes. El tamaño de la muestra se obtuvo mediante la ecuación estadística para poblaciones finitas (ecuación 1), asumiendo un nivel de confianza del $95 \%$ y un margen de error del $5 \%$, así como unas variabilidades tanto positivas como negativas del $50 \%$, respectivamente (Martínez, 2005):

$$
n=\frac{Z^{2} p q N}{\left(E^{2}(N-1)+p q Z^{2}\right)}
$$

En esta ecuación,

$\mathrm{n}=$ Tamaño de la Muestra.

$$
\begin{aligned}
& Z=\text { Nivel de Confianza } 95 \%=1,96 \\
& p=\text { Variabilidad Negativa }=50 \% \\
& E=\text { Error }=5 \%=0.05
\end{aligned}
$$

$q=$ Variabilidad Positiva $=50 \%$

Reemplazando estos datos en la ecuación 1, se obtuvo los siguientes resultados:

$$
n=\frac{(1,96)^{2}(0,5)(0,5)(440)}{\left((0,05)^{2}(440-1)+(0,5)(0,5)(1,96)^{2}\right)}=205,34
$$

Definido el tamaño de la muestra, se aplicaron 205 encuestas aleatoriamente entre los clientes del restaurante en referencia, quienes a través de una escala de Likert de 1 a 7 , indicaron sus percepciones respecto a cada uno de las veintidós declaraciones de las que se alimentan las cinco dimensiones del modelo ServQual

\begin{tabular}{|c|c|}
\hline Dimensión & Ítems de Evaluación por Dimensión \\
\hline \multirow{4}{*}{$\begin{array}{l}\text { Elementos } \\
\text { Tangibles - ET }\end{array}$} & ET 1. Los equipos que emplea el servicio tienen apariencia moderna. \\
\hline & ET 2. Las instalaciones físicas del servicio tienen apariencia moderna. \\
\hline & ET 3. Los empleados del servicio tienen apariencia pulcra. \\
\hline & ET 4. Los materiales relacionados con el servicio (la carta, publicidad) son Visualmente atractivos. \\
\hline \multirow{5}{*}{ Fiabilidad - F } & F 5. Cuando el servicio promete hacer algo en cierto tiempo realmente lo hace. \\
\hline & F 6. Cuando tengo algún problema con el pedido, el servicio muestra interés en resolverlo. \\
\hline & $\begin{array}{l}\text { F 7. El servicio ofrecido por restaurante en la ciudad de Santa Marta - Colombia, ha realizado bien } \\
\text { su atención desde la primera vez. }\end{array}$ \\
\hline & F 8. Se cometen errores con los registros y anotaciones de los pedidos. \\
\hline & F 9. El servicio concluye la atención en el tiempo prometido o estipulado por sus meseros. \\
\hline \multirow{4}{*}{$\begin{array}{l}\text { Capacidad de } \\
\text { Respuesta - CR }\end{array}$} & CR 10. Los empleados comunican cuando concluirá el servicio. \\
\hline & CR 11. Los empleados ofrecen un servicio rápido. \\
\hline & CR 12. Los empleados están dispuestos a ayudar. \\
\hline & CR 13. Los empleados responden correctamente las preguntas que se les hacen. \\
\hline \multirow{4}{*}{ Seguridad - S } & S 14. El comportamiento de los empleados transmite confianza. \\
\hline & S 15. Me siento seguro/a con el servicio. \\
\hline & S 16. Los empleados son amables. \\
\hline & S 17. Se siente seguro al momento de cancelar el servicio ya sea efectivo o con tarjeta. \\
\hline \multirow{5}{*}{ Empatía - E } & E 18. Recibo atención individualizada. \\
\hline & E 19. El horario de la prestación del servicio me resulta cómodo. \\
\hline & E 20. El servicio se preocupa por mis intereses, y por lo que como cliente pueda desear. \\
\hline & E 21. Los empleados comprenden mis necesidades. \\
\hline & 2. Los empleados tienen conocimientos para resp \\
\hline
\end{tabular}
(elementos tangibles, fiabilidad, capacidad de respuesta, seguridad y empatía), tal como se muestra en la Tabla 2. A partir de este instrumento, los clientes manifestaron las valoraciones tanto de sus expectativas respecto al servicio que esperaban recibir, como del servicio recibido efectivamente.

Tabla 2: Dimensiones e Ítems del Modelo ServQual Aplicados 
Basado en las valoraciones reveladas por los consumidores respecto a sus percepciones de la calidad del servicio, se determinaron las brechas existentes en cada uno de los renglones del modelo, y se determinó en qué dimensiones la organización se desempeña correctamente a fin de suplir las expectativas del cliente, y en cuáles existen falencias, siendo así necesario implementar medidas para mejorarlas. Posteriormente, se aplicó la Red de Petri, con el objeto de evaluar la capacidad en el ofrecimiento del servicio y el uso eficiente de los recursos asignados por parte del Restaurante, de acuerdo a los resultados encontrados en la tercera dimensión del ServQual, es decir, capacidad de respuesta. Para lograr esto se procedió con la recopilación de información y análisis de datos de tiempos de toma de pedidos, tiempos de elaboración de pedidos, tiempos de atención de pedidos a domicilios y tiempos de llegadas de clientes al restaurante. La respectiva simulación de la Red de Petri, se hizo mediante el uso del lenguaje Visual Basic, a fin de simular el servicio de atención al cliente en el restaurante de la ciudad de Santa Marta - Colombia. Además, para poder realizar una mejora fue necesario diagnosticar la situación actual del sistema y las limitaciones que presenta, con el propósito de poder empezar a corregir los puntos que generen la problemática presentada.

En tal sentido, es de destacar que el servicio de atención en el Restaurante, presenta dos elementos fundamentales que con su interacción se encargan de estructurar el servicio como lo son: la toma de pedidos y su proceso de elaboración. Además, también es de resaltar que los domicilios a pesar de no ser un servicio que se refleje en cuanto a una atención presencial observable a simple vista, también componen un eje importante, debido a que ocupan tiempo en la elaboración de alimentos y recursos a la hora de incluir_una visión totalmente real del servicio. Así las cosas, se procedió con diversas visitas al local durante los horarios de operación, y por periodos de una hora se tomaron los tiempos de atención de los meseros. Al momento de realizar el estudio, el restaurante contaba con 3 meseros encargados de prestar el servicio. Para analizar los datos, se hizo uso de dos herramientas informáticas: Input Analyser y Statgraphics. En el caso de los pedidos a domicilio, se hizo necesario pedir al administrador del restaurante los registros de los mismos, a lo cual se pudo acceder y se tomó una muestra de un total de 55 domicilios, en un periodo de una semana. Debido a que se contó con los horarios en los cuales sucedieron, se pudo contabilizar el intervalo de tiempo que pasaba entre la realización de un pedido y otro.

Por último, se tomaron los datos de llegada de clientes, mediante intervalos de tiempo entre dos llegadas sucesivas de clientes. Es de anotar que esta variable tiene un comportamiento más complejo y comprometedor para el sistema, debido a que los clientes llegan en un determinado tiempo, pero esto no quiere decir que se queden en el restaurante, ya que una vez que se ha alcanzado la capacidad del restaurante (el restaurante está totalmente ocupado), es imposible para los dueños del negocio ofrecerles el servicio en instalaciones secundarias o abrir nuevo campo para ellos, con lo que los clientes desisten del servicio y se retiran del local. Actualmente la capacidad del local se ubica en 10 pedidos simultáneos, eso quiere decir que si en determinado momento existe una cola de espera que iguale este número, los clientes en general tenderán a abandonar el lugar, con lo que se tendrá una perdida, que no solo se ve desde el punto de vista monetario, sino también de la imagen del Restaurante.

\section{RESULTADOS}

Realizadas todos las etapas metodológicas y aplicadas todas las técnicas previstas, se obtuvieron los resultados del estudio. En primera instancia, se hallaron las brechas entre percepción y expectativas para cada una de las dimensiones del ServQual, indicando los déficits de calidad del servicio cuando la puntuación de expectativas supere a la de percepción, tal como se observa en la Tabla 3.

Tabla 3: Brechas por Dimensiones y preguntas del Modelo ServQual en restaurante de la ciudad de Santa Marta

\begin{tabular}{|l|c|c|c|c|c|}
\hline \multirow{2}{*}{\multicolumn{1}{|c|}{ Dimensiones }} & \multicolumn{2}{c|}{ Valor Esperado } & \multicolumn{2}{c|}{ Valor Percibido } & Brecha \\
\cline { 2 - 6 } & Promedio & Desv. estándar & Promedio & Desv. estándar & $P-E$ \\
\hline Elementos Tangibles - ET & 6,78 & 0,45 & 6,15 & 0,59 & $(-0,63)$ \\
\hline Fiabilidad - F & 5,86 & 0,60 & 3,96 & 1,10 & $(-1,90)$ \\
\hline Capacidad de Respuesta - CR & 6,51 & 0,57 & 4,16 & 1,38 & $(-2,35)$ \\
\hline Seguridad - S & 6,28 & 0,48 & 6,18 & 0,32 & $(-0,10)$ \\
\hline Empatía - E & 5,70 & 0,37 & 5,38 & 0,86 & $(-0,32)$ \\
\hline
\end{tabular}

Los resultados revelan que las mayores brechas entre expectativas y percepciones de la calidad del servicio del Restaurante objeto de estudio, se hallaron en dos dimensiones que tienen alta relación por su implicancia en cuanto a la prestación del servicio ofrecido, estas fueron capacidad de respuesta y fiabilidad. Es decir, los clientes esperaban más a cerca de su experiencia en el Restaurante de lo que realmente recibieron o percibieron en estas dimensiones. Esto es un indicador clave respecto a las falencias del servicio que está 
presentando esta organización y por lo tanto, se debe prestar atención para trabajar en ellas e incrementar los niveles de satisfacción de los clientes.

En tal sentido, la dimensión capacidad de respuesta, presentó la brecha más significativa, dado que los resultados muestran que el personal no logra responder con prontitud y habilidad a estos requerimientos, razón por la cual los clientes se encuentran insatisfechos. Por tal motivo, hay dos aspectos que requieren atención inmediata; que consiste, en que los empleados no están comunicando cuando concluirá el servicio y no están brindando un servicio con prontitud y eficacia al cliente, reflejando una importante deficiencia, por tanto es necesario disminuir el tiempo de espera y el personal debe trabajar de forma más coordinada al presentarse estas situaciones (Ver Figura 1).

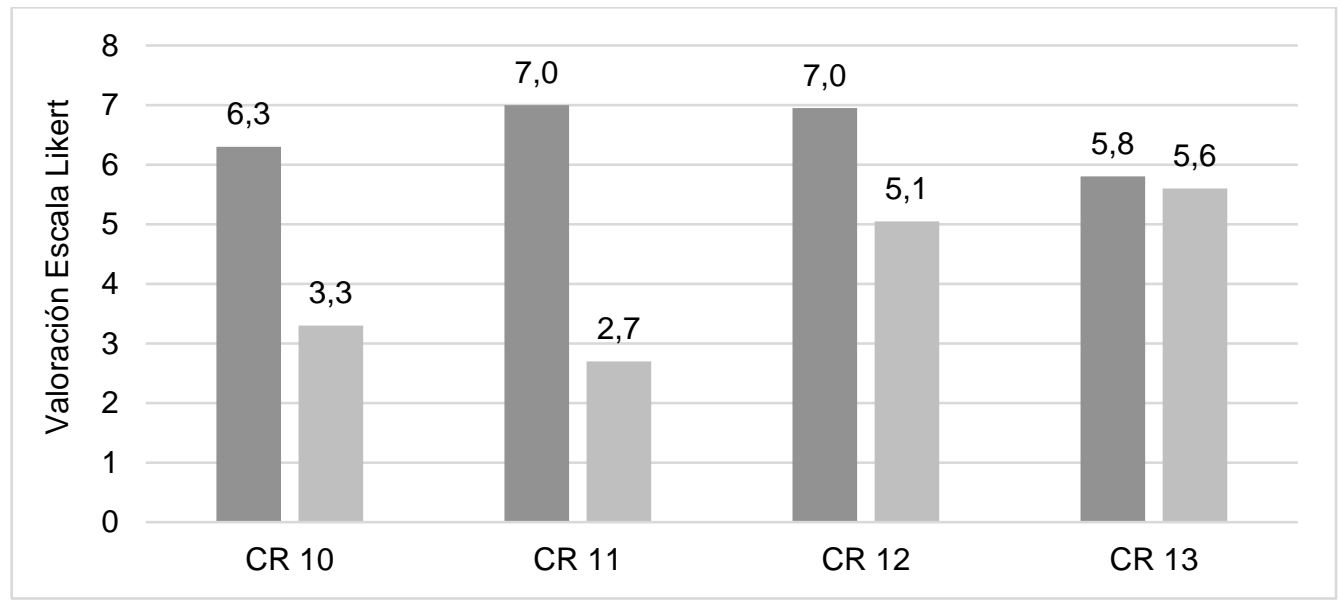

Fig. 1: Brecha de la Dimensión Capacidad de Respuesta

Por su parte, la Fiabilidad es la dimensión con la segunda brecha más significativa, al contrastar las expectativas y percepciones develadas por los clientes. Por ello, es necesario que la administración del Restaurante trabaje fuertemente en mejorar cada uno de los aspectos que hacen parte de esta dimensión, especialmente cumplirle a los clientes sobre la entrega de pedidos en los tiempos establecidos, y mantenerles informados sobre los momentos en los cuales se efectuara efectivamente el servicio solicitado (ver Figura 2), dado que en gran medida, de ello dependerá la confianza del cliente respecto al cumplimiento de la promesa de venta hecha por el restaurante, al segmento al que se dirige.

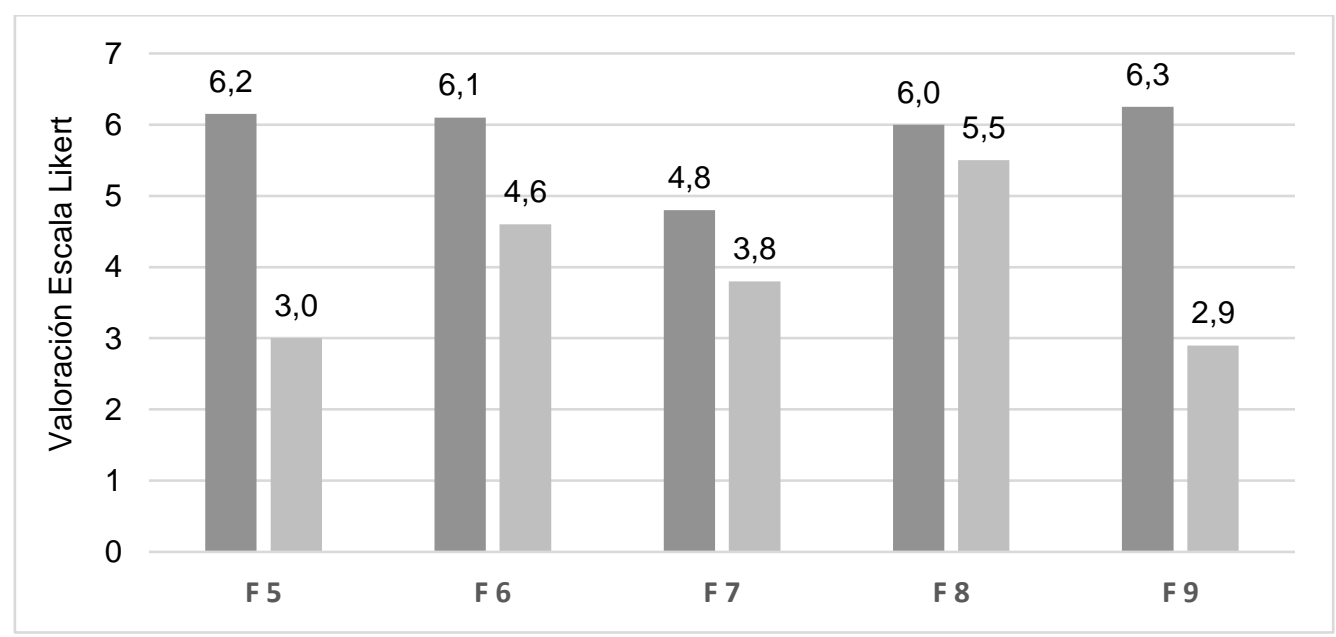

Fig. 2: Brecha de la Dimensión Fiabilidad

De igual manera, se evidenció que el problema de la calidad del servicio del Restaurante, radica en una importante deficiencia en los tiempos de espera y de atención al cliente, resultando necesario disminuir estos tiempos de cola (tiempo de toma de pedidos, tiempos de elaboración, tiempos de domicilios) así como entender la dinámica de acceso de los clientes al restaurante. Consecuente con esta problemática, se procedió mediante las herramientas Input Analyser y Statgraphics con el fin de poder conocer el tipo de distribución de los datos muestreados, y con ello poder plantear con suficiente certeza inferencias del comportamiento poblacional. 
Además de lo anterior, se aplicó a dichos tiempos la prueba de bondad de ajuste de Kolmogorov-Smirnov (dado que se manejan variables aleatorias continuas), con el fin de contrastar si los datos de la muestra se consideraban que procedían de determinada distribución. Ratificando para este caso, que los tiempos de toma de pedidos y de pedidos a domicilio se ajustan a una distribución normal, los tiempos de elaboración a una distribución uniforme, y los tiempos de llegada de clientes se ajustan a una distribución exponencial (Ver Tabla 4). Por último, se describe y simula la situación actual y la situación propuesta en Red de Petri.

Tabla 4: Distribución de Datos muéstrales de los tiempos de toma de pedidos, elaboración, domicilios y llegada de clientes al Restaurante de la Ciudad de Santa Marta - Colombia

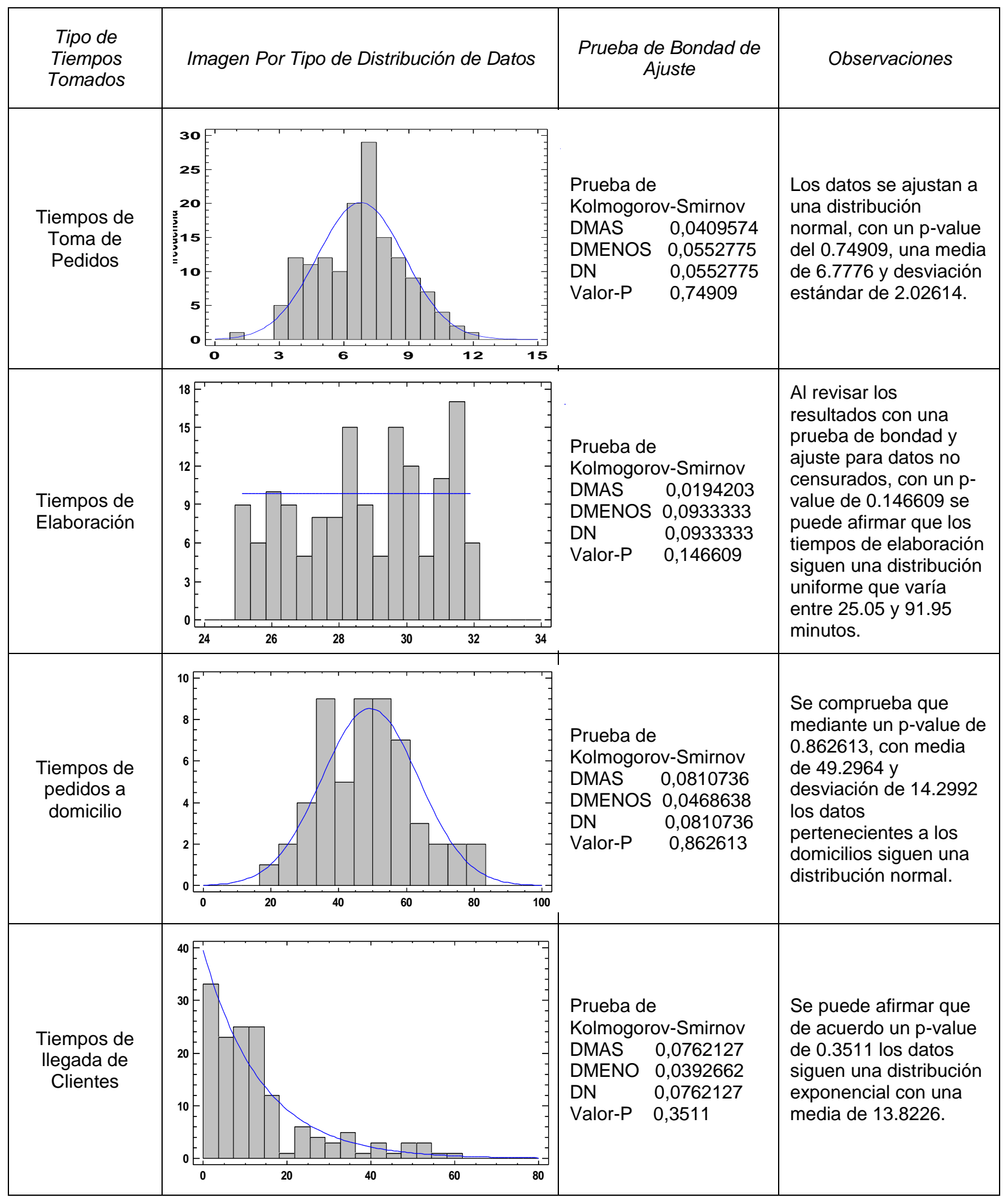




\section{Situación Inicial}

Al momento de realizar la investigación, el restaurante objeto de estudio contaba en su capacidad instalada con un solo cocinero para atender todo los pedidos solicitados por los clientes. Luego de realizar 7 horas de simulación se encontró que en el negocio solo se atendían 11 pedidos, que en cola permanecían 9 pedidos, que los domicilios en cola ascendían a 11, y que se retiraban del restaurante 12 clientes. Estos resultados evidenciaron que el restaurante no contaba con un funcionamiento acorde a lo esperado, debido a que tenía una baja capacidad de recibir pedidos y de estos muchos quedaban pendientes o en cola, por lo que es necesario realizar un cambio en el funcionamiento actual. Para esto se plantea una red de Petri, donde se tienen en cuenta los domicilios y la capacidad del local (Ver Tabla 5 y Figura 4).

Tabla 5: Declaración de variables de conformación de la Red de Petri situación inicial para la prestación del servicio en el restaurante de la Ciudad de Santa Marta - Colombia.

\begin{tabular}{|l|l|}
\hline T1: Llegada clientes & P8: Disponibilidad mesero 3 \\
\hline P1: Sitio llegada clientes & P9: Mesero atendiendo \\
\hline T2: Comienzo clientes entrada local & T9: Finalización atención mesero 3 \\
\hline T3: Comienzo clientes retirada del local & P10: Ordenes en Espera \\
\hline P2: Clientes en el local & T10: Llegada domicilios \\
\hline P3: Clientes que se retiran del local & P11: Domicilios en espera \\
\hline T4: Comienzo atención mesero 1 & T11: Comienzo atención domicilios \\
\hline P4: Disponibilidad mesero 1 & P12: Cocinero atendiendo domicilios \\
\hline P5: Mesero atendido & P13: Disponibilidad cocinero \\
\hline T7: Finalización atención mesero & T13: Finalización atención cocinero pedidos \\
\hline T5: Comienzo atención mesero 1 & T12: Comienzo atención cocinero ordenes \\
\hline P6: Disponibilidad mesero 2 & P14: Cocinero atendiendo pedidos \\
\hline P7: Mesero atendiendo & T14: Finalización atención cocinero ordenes \\
\hline T8: Finalización atención mesero 2 & P15: Ordenes totales atendidas \\
\hline T6: Comienzo atención mesero 3 & \\
\hline
\end{tabular}

Complementario a la declaración de las variables que conforman la Red de Petri, en la figura 3, se muestra la forma gráfica en la que interactúan las solicitudes, los tiempos de llegada y demás dinámicas del negocio inherentes a su proceso de calidad del servicio, describiendo la situación inicial del restaurante, tal como sucede de manera espontánea, antes de la simulación e intervención propuesta por los investigadores.

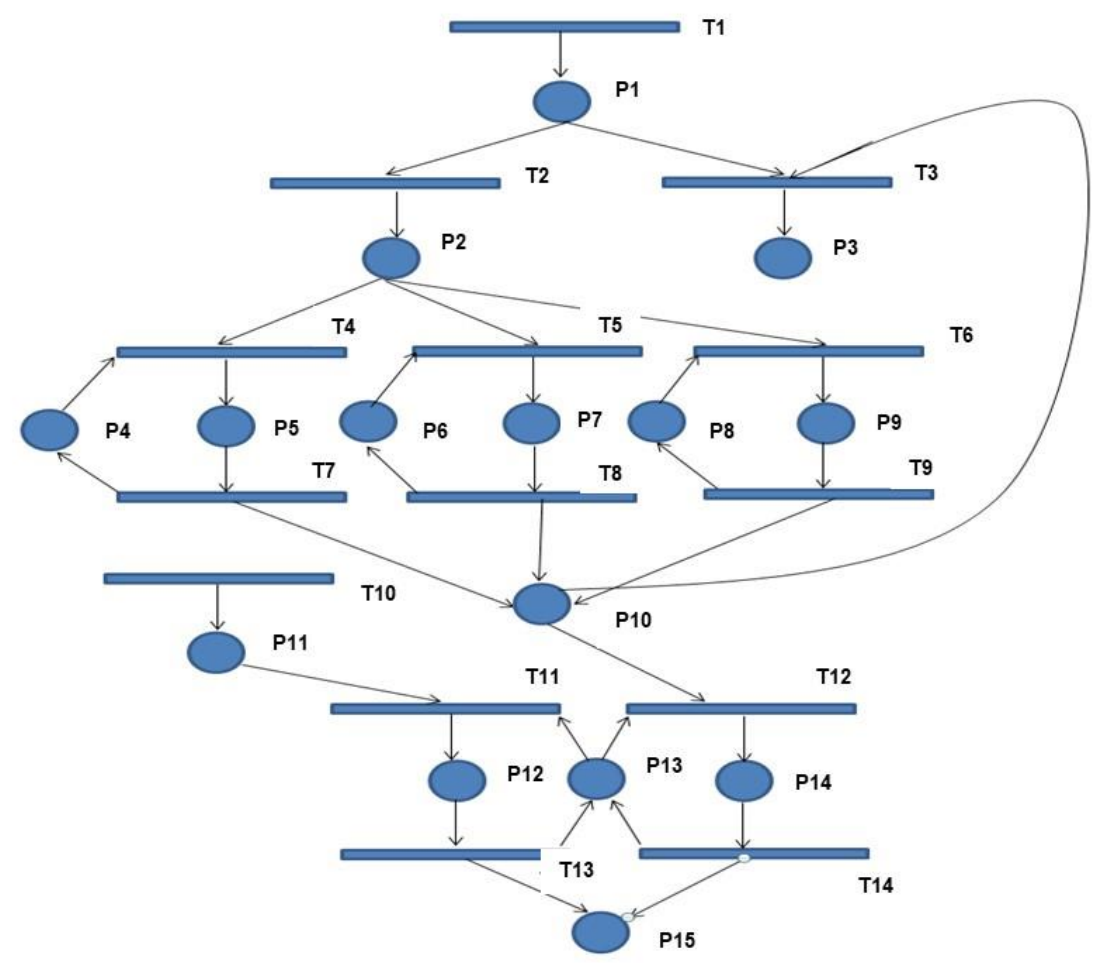

Fig. 3: Simulación Situación Actual en Red de Petri. 


\section{Situación Propuesta}

Luego de observar los resultados presentados en la primera simulación, se procedió a realizar una mejora en el recurso crítico del proceso, que consistía fundamentalmente en el cocinero. En tal sentido, se procedió a simular una situación con un segundo cocinero, incrementándose el número de pedidos atendidos a 25 y disminuyendo la cantidad de pedidos en cola a solo 3. De igual manera, también se redujo el número de domicilios en cola a 9 y el número de clientes que se retiraron del restaurante terminó siendo cero. Con esta nueva mejora se pudo observar un aumento sustancial de los pedidos atendidos, lo cual a su vez terminó representando un incremento en las ventas realizadas. Por lo tanto, la opción de poner un segundo cocinero hará que las colas en la elaboración de los pedidos disminuyan. Sin embargo se pudo observar que los domicilios continúan representando un problema a tratar dentro del sistema, dado que siguen implicando retrasos e inconformismos; producto de ello, será necesario establecer estrategias futuras que posibiliten solventar dicha situación de manera más eficiente (Ver Tabla 6 y Figura 5).

Tabla 6: Propuesta de Variables de Conformación de Nueva Red de Petri para la prestación del servicio en el restaurante de la Ciudad de Santa Marta - Colombia

\begin{tabular}{|l|l|}
\hline T1: Llegada clientes & P10: Ordenes en Espera \\
\hline P1: Sitio llegada clientes & T10: Llegada domicilios \\
\hline T2: Comienzo clientes entrada local & P11: Domicilios en espera \\
\hline T3: Comienzo clientes retirada del local & T11: Comienzo atención domicilios cocinero 1 \\
\hline P2: Clientes en el local & P12: Cocinero 1 atendiendo domicilios \\
\hline P3: Clientes que se retiran del local & P13: Disponibilidad cocinero 1 \\
\hline T4: Comienzo atención mesero 1 & T15: Finalización atención cocinero 1 pedidos \\
\hline P4: Disponibilidad mesero 1 & T12: Comienzo atención cocinero 1 ordenes \\
\hline P5: Mesero atendiendo & P14: Cocinero 1 atendiendo ordenes \\
\hline T7: Finalización atención mesero & T16: Finalización atención cocinero 1 ordenes \\
\hline T5: Comienzo atención mesero 1 & T12: Comienzo atención domicilios cocinero 2 \\
\hline P6: Disponibilidad mesero 2 & P15: Cocinero 2 atendiendo domicilios \\
\hline P7: Mesero atendiendo & P16: Disponibilidad cocinero 2 \\
\hline T8: Finalización atención mesero 2 & T17: Finalización atención cocinero 2 domicilios \\
\hline T6: Comienzo atención mesero 3 & T14: Comienzo atención cocinero 2 ordenes \\
\hline P8: Disponibilidad mesero 3 & P17: Cocinero 2 atendiendo ordenes \\
\hline P9: Mesero atendiendo & T18: Finalización atención cocinero 1 ordenes \\
\hline T9: Finalización atención mesero 3 & P18: Ordenes totales atendidas \\
\hline
\end{tabular}

Al igual que en el escenario de la situación inicial (tabla 5 y figura 3), seguidamente la figura 4 presenta la forma gráfica en la que interactúan las dinámicas del restaurante objeto de estudio inherentes a su proceso de calidad del servicio, describiendo la situación propuesta, mediada por la simulación e intervención planteada por los investigadores que realizaron este estudio.

Mediante el análisis de los resultados obtenidos, se pudo observar distintas falencias del restaurante objeto de estudio, que pueden ser solventadas mediante la mejora de los recursos existentes. En este punto, surge la implementación de la mejora continua como alternativa de solución incremental, ya que el servicio debe ser cada vez más apropiado y congruente con las expectativas del cliente. En consecuencia, en la sucursal del restaurante de la ciudad de Santa Marta, se deben adoptar estrategias que permitan solventar las necesidades y expectativas de los clientes a cabalidad; tal como lo manifiestan Daza et al (2015), y Raajpoot (2004), quien explica que la percepción de sentirse "bien atendido" responde, en muchos casos, a elementos intangibles de atención rápida, tendiendo hasta cierto punto a conformar concepciones particulares de lo que se entiende por calidad en el servicio. Además de lo anterior, a través de los resultados hallados en esta investigación se ratifica lo expuesto por Guzman y Carcamo (2014), quienes argumentan la existencia de una influencia significativa de la calidad del servicio en la satisfacción del consumidor, la cual además influye significativamente en su lealtad de compra y la recomendación del negocio a terceros cercanos. De igual manera, resultó útil acudir a la base de estudios de teoría de colas y de herramientas de simulación apoyadas por software estadístico, a fin de facilitar soluciones a los distintos problemas del sistema y aplicar una solución factible, en este caso la contratación del segundo cocinero; concordando así con lo expuesto por Moon (2013), quien argumenta que el método de Red de Petri presenta el potencial de generar conocimiento sobre los procesos de producción en la prestación de servicios, implicando una nueva relación proveedor-cliente para las acciones de mejora. 


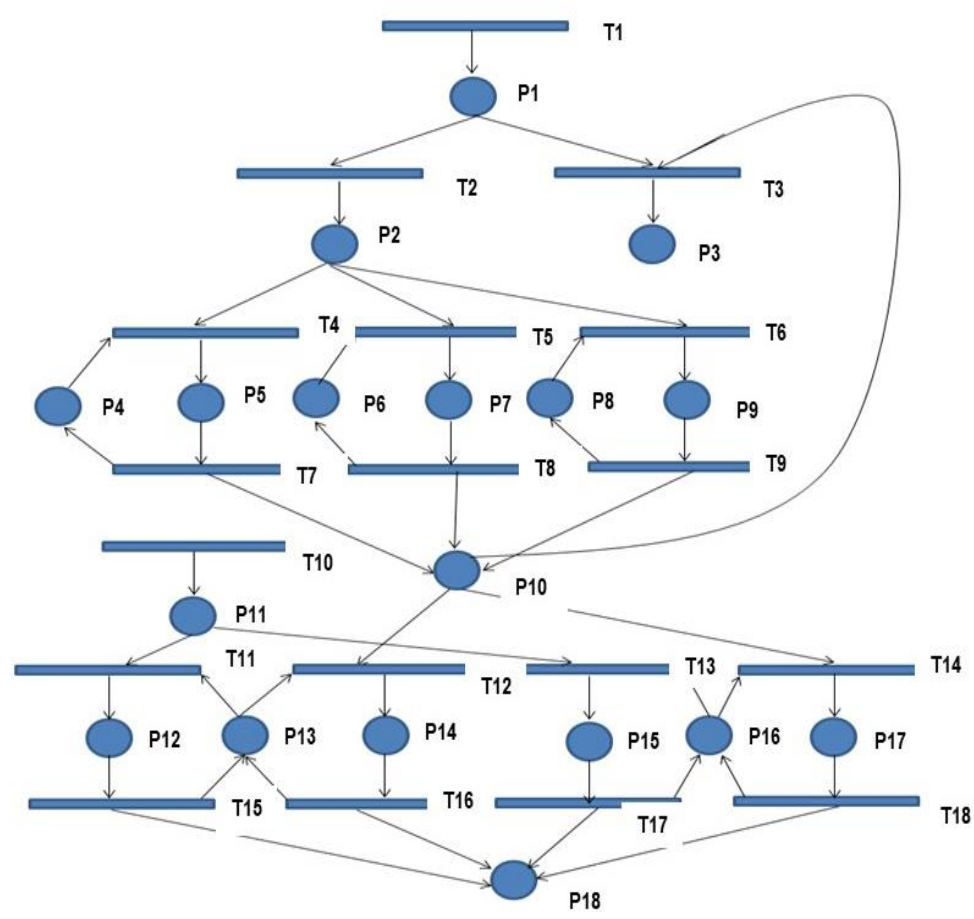

Fig. 4: Simulación Situación Propuesta del modelo de Red de Petri

Finalmente es de notar que los tiempos de llegada y de elaboración, tienen una diferencia considerable, lo cual permite observar a simple vista que el sistema puede en algún momento no ser capaz de cumplir con la demanda esperada, lo cual converge con los resultados obtenidos en su momento por Sheffi y Rice (2005). Por lo tanto, se hace necesario mejorar las dimensiones de la calidad del servicio con falencias, que en este caso no están relacionadas con el proceso de elaborar los productos, debido a que estos tienen una buena calidad, el mayor problema radica en la imposibilidad de brindar los alimentos en ciclo de tiempo concurrente con las expectativas del cliente. Esto sucede debido a que el sitio de preparación de alimentos se ve limitado a un solo operario, además de eso su puesto de trabajo no es lo suficientemente amplio por lo que solamente puede realizar un limitado número de pedidos al mismo tiempo; justificando así la necesidad de continuar con más estudios de mejora continua que aporten nuevas soluciones y demás medidas a implementar.

\section{DISCUSION FINAL}

Mediante la aplicación de la herramienta del Servqual en el presente estudio, se puede establecer que es posible determinar el nivel de la calidad de un servicio, sobre todo en el caso de servicio de atención en un restaurante, basados en los supuestos de abstracción y dimensionalidad; lo cual implica una serie de posiciones tanto teóricas como empíricas (Guzmán y Cárcamo, 2014). Razón por la cual, lo verdaderamente determinante de esta herramienta, es su validez para medir mediante escalas de abstracción las realidades en la prestación de un servicio, versus lo ideal de la obtención del servicio de manera estandarizada, permitiendo de esta manera, realizar comparaciones de satisfacción alcanzadas por los distintos usuarios de un servicio en un momento determinado, cruzando además las distintas percepciones de manera racional mediante la examinación de la dimensionalidad, así como la estabilidad de las dimensiones subyacentes (Du y Tang, 2014).

Sin embargo, autores como Mc Collin et al (2011), Laguna y Palacios (2008), Cook et al (1999) y Babakus y Boller (1992), entre otros, se han encargado de examinar la viabilidad de definir operacionalmente la calidad del servicio en términos de brechas de expectativa/percepción, evaluación de los efectos de la redacción del elemento (es decir, connotación negativa frente a positiva) en la calidad de la respuesta y los resultados del análisis factorial, identificando problemas de confiabilidad y validez del modelo Servqual con implicaciones determinantes para el desarrollo teórico y la medición en los servicios del área de marketing en cualquier organización, lo cual es subyacente a las herramientas o modelos que pretendan explicar la realidad de un hecho. En este marco, en el caso de la calidad del servicio en restaurantes, autores como Cabana et al (2015), y Fuentes-Blasco y Moliner-Velásquez (2014), manifiestan que los clientes de cualquier tipo de mercado y sobre todo del servicio de restaurantes, cada día demandan a la administración de servicios de mayor calidad.

Quieren servicios más rápidos y fiables, con horarios más amplios, servidos por funcionarios que les tratan con cortesía y respeto; lo cual obliga a la gerencia del Restaurante a entregar a sus clientes servicios de 
calidad adaptados a sus necesidades y expectativas. Por lo tanto, el factor clave para lograr un alto nivel de calidad en el servicio de este restaurante, es igualar o sobrepasar las expectativas que el cliente tiene respecto al servicio recibido; para lo cual, el modelo Servqual y de las redes de Petri han de facilitar información veraz a las decisiones acertadas en nuevos marcos de acción en la prestación de servicios de calidad.

En este sentido, se pudo evidenciar que en el restaurante de la ciudad de Santa Marta, las percepciones de los clientes presentan grandes falencias en las dimensiones de fiabilidad y capacidad de respuesta, por tanto es necesario trabajar de forma inmediata en el mejoramiento continuo de estas dimensiones. Por otra parte, se evidencio que las dimensiones con la mejor percepción por parte del cliente, son elementos tangibles y seguridad. Además, se proponen recomendaciones como restringir el acceso temporal por parte del cliente al restaurante cuando se encuentre lleno, para no causar inconformidades con respecto al tiempo de espera; analizar si la cantidad de mesas disponibles en la actualidad cumplen de manera eficiente con la demanda existente, los días que la capacidad del restaurante alcance su límite, y entregar un formato de encuesta de satisfacción al cliente consumidor al momento de finalizar el servicio.

\section{CONCLUSIONES}

De acuerdo al trabajo presentado y a los resultados obtenidos, se pueden plantear las siguientes conclusiones principales:

1) Las dos herramientas implementadas en el restaurante objeto de estudio (ServQual y Red de Petri), permitieron evaluar la calidad en la prestación del servicio e influir en la satisfacción del cliente, garantizando su fidelización y la sostenibilidad de la organización en el mercado.

2) Obtención de mejora de la calidad del servicio del restaurante estudiado, consecuencia del incremento de la capacidad de pedidos atendidos (25), disminución de pedidos en cola (solo 3), así como del número de domicilios en cola (9) y cero clientes retirados del restaurante por no ser atendidos a tiempo.

3) El aporte fundamental de este artículo, consiste en la aplicación conjunta y sinérgica de una herramienta de diagnóstico de la calidad del servicio (Servqual) y la experimentación de escenarios mediante la representación gráfica y matemática de un sistema a eventos (Red de Petri), para optimización de procesos en la prestación del servicio, en contraste con los estudios realizados en otros ámbitos, que solo aplican una de una de las dos herramientas, más no ambas simultáneamente.

4) El desconocimiento de los propietarios y la gerencia del restaurante respecto a conceptos, herramientas y técnicas de servicio al cliente y sistema de eventos, tales como Servqual, Red de Petri, entre otros, limitaba el desempeño operativo, la eficiencia y la eficacia del negocio, lo cual devela la necesidad de cualificación en esta materia.

\section{REFERENCIAS}

Adam, N.R., V. Atluri y W.K. Huang, Analysis of Workflows Using Petri Nets, Journal of Intelligent Information Systems, 10, 131-158 (1998)

Babakus, E. y G. Boller, An Empirical Assessment of the ServQual Scale, Journal of Business Research, 24, 253-268 (1992)

Cabana, S. R., P. A. Gálvez y C. I. Muñoz, Variables Críticas en las Ventajas Competitivas de Restaurantes Gourmet, La Serena, Chile, Cuadernos de Administración Universidad del Valle, 31(54), 57-67 (2015)

Callejas-Cuervo, M., H. Valero-Bustos y A. Alarcón-Aldana, Agentes de Software como Herramienta para Medir la Calidad de Servicio Prestado en un Sistema de Transporte Público Colectivo Urbano, Información Tecnológica, 25 (5), 147 - 154 (2014)

Causado-Rodríguez, E., R.L. Díaz y M. R. Rago, Avances del Proceso de Calidad en la Prestación de los Servicios de Salud en Colombia, Revista Duazary, 5(2), 161-166 (2008)

Causado-Rodríguez E., J. García, J. Martínez y A. Herrera, Tecnologías de Información y Comunicación en el Sector Hotelero, Barranquilla, Edit. Corporación Universitaria Latinoamericana CUL, 118, 15 x 20 (2015)

Cook, D.P., G. Chon-Huat y H.C.Chung, Service Typologies: A State of the Art Survey, Production and Operations Management, 318 (1999)

Daza, A., A. Charris y J. Viloria, Competencias Específica de los Administradores Como Factor de Desarrollo, Dimensión Empresarial, 13 (2), 275 - 292 (2015)

Du, Y. e Y. Tang, A Literature Review on the Relationship Between Service Quality and Customer Loyalty, Business and Management Research, 3(3), 27-33 (2014)

Fuentes-Blasco, M. y B. Moliner-Velázquez, Antecedentes del Éxito de la Relación entre Restaurantes y sus Clientes, Innovar, 24(53), 99-112 (2014) 
Guzman, A. y M. Carcamo, La Evaluación de la Calidad en el Servicio. Caso de Estudio Restaurante Familiar los Fresnos, Acta Universitaria, 24(3), 35 - 49 (2014)

Jensen, K., Coloured Petri Nets. Basic Concepts, Analysis Methods and Practical Use; EATCS Monographs on Theoretical Computer Science, Springer-Verlag, Berlin (1996)

Kim, D., J.P. Leigh, Are Meals at Full-Service and Fast-Food Restaurants "Normal" or "Inferior"? Popul. Health Manag., 14 (6), 307-315 (2011)

Laguna, M. y A. Palacios, La Calidad Percibida como Determinante de Tipologías de Clientes y su Relación con la Satisfacción: Aplicación a los Servicios Hoteleros, Revista Europea de Dirección y Economía de la Empresa, 18(3), 189210 (2008)

Law, A.K., Y.V. Hui y X. Zhao, Modeling Repurchase Frequency and Customer Satisfaction for Fast Food Outlets, Int. J. Qual. Reliab. Manag. 21 (5), 545-563 (2004)

Lee, Y.L. y N. Hing, Measuring Quality in Restaurant Operations: an Application of the Servqual Instrument, Int. J. Hospitality Management, 14 (3/4), 293-310 (1995)

Liu, Ch, Petri Nets Model for Service Engineering, Proceedings of the IEEE International Conference on Automation and Logistics, Shenyang, China August, 2009, 632 - 637 (2009)

López, C. y A. Serrano, Dimensiones y Medición de la Calidad de Servicio en Empresas Hoteleras, Revista Colombiana de Marketing, 2(3), 1-13 (2001)

McCollin, Ch, I. Ograjenšek, R. Göb y A. Ahlemeyer-Stubbed, SERVQUAL and the Process Improvement Challenge, Quality and Reliability Engineering International, 27, 705 - 718 (2011)

Moon, Y., The Tangibility and Intangibility of e-Service Quality, International Journal of Smart Home, 7(5), 91-102 (2013)

Parasuraman, A., V.A. Zeithaml y LL.A. Berry, Conceptual Model of Service Quality and its Implications for Future Research, Journal of Marketing, 49, 41 - 50 (1985)

Parasuraman, A., V.A. Zeithaml y LL.A. Berry, SERVQUAL: A Multiple-Ítem Scale for Measuring Customer Perceptions of Service Quality, Journal of Retailing, 64, 12 - 40 (1988)

Parasuraman, A., LL. A. Berry y V.A. Zeithaml, Refinement and Reassessment of the SERVQUAL Scale, Journal of Retailing, 67, $420-450$ (1991)

Parasuraman, A., V.A. Zeithaml y LL.A. Berry, Reassessment of Expectations as a Comparison Standard in Measuring Service Quality: Implications for Further Research, Journal of Marketing, 58, 111 - 124 (1994)

Petri, C.A., Kommunikation mit Automaten. PhD thesis, Institut für instrumentelle Mathematik, Bonn (1962)

Raajpoot, N., Reconceptualizing Service Encounter Quality in a Non-Western Contex, Journal of Service Research (JSR), 7(2), 181-201 (2004)

Rojas-Ríos, L., J. López-Lezama y N. Muñoz-Galeano, Asignación Óptima de Presupuesto para Mejoramiento de la Calidad del Servicio en Sistemas de Distribución usando Algoritmo Genético No-Dominado II (NSGA-II) y un Algoritmo Memético, Información Tecnológica, 27(1), 115-126 (2016)

Sheffi, Y. y J.B. Rice, A Supply Chain View of the Resilient Enterprise, MIT Sloan Management Review, 47(1), 41-48 (2005)

Silva, V., F. Schramm, M. Costa y A. Danielle, Proposal of a Procedure for Evaluating Individual's Expectations and Perceptions Based on ServQual 2014 IEEE International Conference on Systems, Man, and Cybernetics, San Diego, CA, USA October 5-8 (2014)

Schmal, R. y T. Olave, Optimización del Proceso de Atención al Cliente en un Restaurante Durante Períodos de Alta Demanda, Información Tecnológica, 25(4), 27 - 34 (2014)

Tan, W., Y. Fan y Z. MengChu, A Petri Net-Based Method for Compatibility Analysis and Composition of Web Services in Business Process Execution Language, IEEE Transactions on Automation Science and Engineering, 6, (2) (2009)

Teas, R.K., Consumer Expectations and the Measurement of Perceived Service Quality, Journal of Professional Services Marketing, 8, 33-54 (1993)

Van Der Aalst, W.M.P, Putting Petri Nets to Work in Industry, Computers in Industry, 25(1), 45-54 (1994)

Yu-qiang, S. y W. Jun-Jia, Evaluation of Service Quality of Restaurant Enterprise Based on ServQual, International Conference on Management and Service Science, 12 (14), 1 - 3 (2011) 